\title{
Permalloy thin films exchange coupled to arrays of cobalt islands
}

\author{
A. Fraile Rodríguez, a) L. J. Heyderman, and F. Nolting \\ Paul Scherrer Institut, 5232 Villigen PSI, Switzerland
}

\author{
A. Hoffmann and J. E. Pearson \\ Material Science Division, Argonne National Laboratory, Argonne, Illinois 60439 \\ L. M. Doeswijk, M. A. F. van den Boogaart, and J. Brugger \\ Microsystems Laboratory, Institute of Microelectronics and Microsystems, \\ École Polytechnique Fédérale de Lausanne (EPFL), 1015 Lausanne, Switzerland
}

(Received 10 April 2006; accepted 2 August 2006; published online 5 October 2006)

\begin{abstract}
Periodic arrays of elongated cobalt islands exchange coupled to continuous Permalloy thin films were fabricated using silicon nitride stencil masks and the magnetic spin configurations during magnetization reversal were studied with photoemission electron microscopy. The presence of cobalt islands results in a spatial modulation of the magnetic properties of the Permalloy films and domain walls positioned at the island boundaries. While magneto-optical Kerr effect measurements indicate differences depending on film thickness, the direct observations reveal two reversal mechanisms: formation of domains running between the islands and coherent rotation followed by propagation of a large domain. (C) 2006 American Institute of Physics. [DOI: 10.1063/1.2357007]
\end{abstract}

Patterned magnetic thin films have been the focus of interest in recent years, not only because of the potential for technological applications, such as magnetic recording, ${ }^{1}$ magnetoresistive random access memory devices, ${ }^{2}$ and logic devices, ${ }^{3}$ but also because of the modified physical behavior as the lateral dimensions are reduced. The functionality of patterned films can be extended by combining different materials in a multilayer stack with the simplest combination being a bilayer comprising two exchange coupled ferromagnetic materials with different magnetic properties. Bilayers with a magnetically hard and a magnetically soft film provide particularly useful model systems ${ }^{4,5}$ for optimization of exchange coupled hard/soft nanocomposites. ${ }^{6}$ Such nanocomposites are often referred to as exchange-spring systems and are promising as high performance magnets because they achieve a high energy product through the combination of a high coercivity in the hard phase and a high saturation magnetization in the soft phase. In addition, hard/soft bilayer systems optimized for high thermal stability and low coercivity could be employed for perpendicular magnetic recording. ${ }^{7}$ In the present study we have extended the concept of a patterned bilayer system, developing a method employing stencil masks to fabricate a continuous soft film exchange coupled to an array of magnetically harder islands that are deposited onto the soft film through the holes of the mask. This results in a spatial modulation of the magnetic properties of the soft continuous film due to the presence of the hard islands, giving a modification of the magnetic spin configuration in the continuous film and providing a means to position domain walls. While other methods can be employed to modulate the properties of a ferromagnetic film, for example, employing ion beam irradiation, ${ }^{8}$ embedded templates, ${ }^{9}$ and surface roughness modulation, ${ }^{10}$ the stencil lithography method has the advantage that it does not destroy the intrinsic properties of the material. We report here on direct observations of the magnetic domain configurations in both ferromagnetic layers employing an x-ray photoemission

${ }^{a)}$ Electronic mail: arantxa.fraile-rodriguez@psi.ch electron microscope (PEEM) and compare the results with hysteresis loops obtained from magneto-optical Kerr effect (MOKE) measurements.

Arrays of cobalt (Co) islands were deposited on continuous Permalloy $(\mathrm{Py}=\mathrm{Ni}[80 \%] \mathrm{Fe}[20 \%])$ thin films by electron-beam evaporation (base pressure $<4 \times 10^{-8}$ Torr) onto silicon wafer substrates [Fig. 1(a)]. The bilayers consisted of Py/Co with thicknesses of $2 \mathrm{~nm}$ Py/2 nm Co, $5 \mathrm{~nm}$ $\mathrm{Py} / 2 \mathrm{~nm} \mathrm{Co}$, and $5 \mathrm{~nm} \mathrm{Py} / 1 \mathrm{~nm}$ Co. Following deposition of the continuous Py layer, a stencil mask with $1 \times 1 \mathrm{~mm}^{2}$ silicon nitride $\left(\mathrm{Si}_{3} \mathrm{Ni}_{4}\right)$ membranes, each containing an 800 $\times 800 \mu \mathrm{m}^{2}$ regular array of holes, ${ }^{11}$ was employed in situ to pattern the Co layers. The stencil mask was placed against the sample surface and the Co layer was subsequently deposited through the holes in the membrane to produce the array of Co islands. The mask was then removed from the surface and a final $1 \mathrm{~nm}$ aluminum cap layer was deposited to prevent oxidation. This fabrication method was chosen because it allows deposition of the continuous, patterned and cap layers without breaking the vacuum, enabling an undisturbed direct exchange coupling between the two ferromagnetic layers. Another possibility is to post-etch the continuous bilayer system but, apart from the difficulty of etching back the up-

(a)

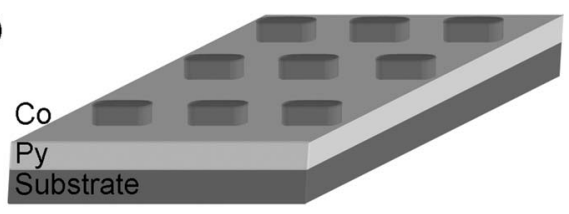

(b)

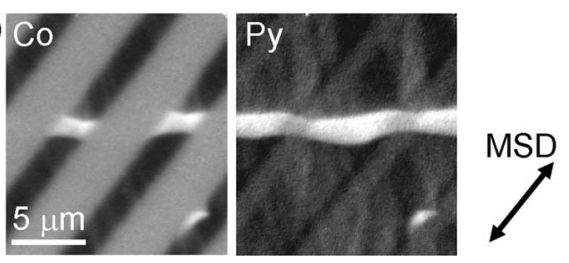

FIG. 1. (a) Schematic diagram of an array of Co islands exchange coupled to a continuous Py thin film. (b) Magnetic spin configuration of a $5 \mathrm{~nm}$ Py/2 nm Co bilayer with $50 \mu \mathrm{m}$-long Co islands. 


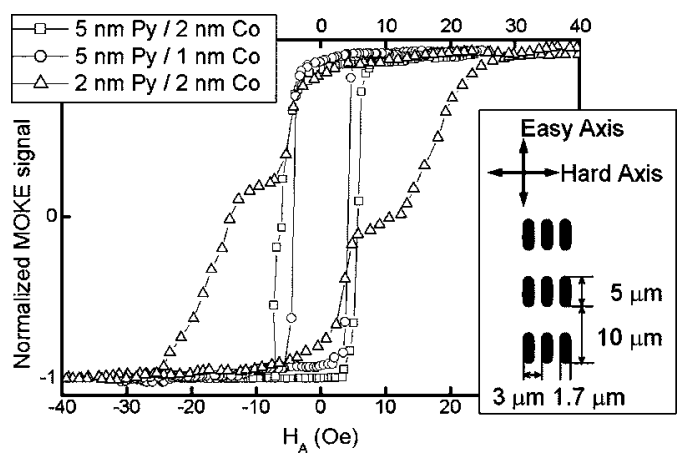

FIG. 2. Easy axis hysteresis loops for Py/Co bilayers with arrays of $5 \mu \mathrm{m}$-long Co islands for different film thicknesses. The inset shows the island geometry.

per Co layer exactly to the Py surface, this would result in unwanted etch damage at the island edges and the film surfaces.

The magnetic imaging was carried out at the SIM beamline at the Swiss Light Source, Paul Scherrer Institut, using an Elmitec PEEM. Employing x-ray magnetic circular dichroism (XMCD), the magnetic spin configurations in both the Co and Py can be observed consecutively by tuning the $\mathrm{x}$-ray energy to the resonant absorption edges of $\mathrm{Co}$ $(778.1 \mathrm{eV})$ and $\mathrm{Ni}(852.7 \mathrm{eV})$, respectively. Dividing two images taken with left and right circular polarized light leads to a so-called XMCD image where the intensity is a measure of the angle between the circular X-ray polarization vector, labeled as the magnetization sensitivity direction (MSD) in the figures, and the magnetic spins in the domains. Ferromagnetic domains with magnetic spins parallel or antiparallel to the polarization vector appear black or white in the XMCD image and domains with magnetic spins perpendicular to the polarization vector will have a grey contrast. While previous PEEM observations have been restricted to measurements at remanence, we were able to observe the magnetic spin configurations in applied magnetic fields up to 100 Oe using a specially designed magnetic sample holder and a recently developed alignment strategy of the microscope.

An example of the magnetic spin configuration observed in a $5 \mathrm{~nm}$ Py/2 nm Co sample with regularly spaced Co islands (length $=50 \mu \mathrm{m}$, width $=1.6 \mu \mathrm{m}$, and period $=5 \mu \mathrm{m}$ ) in the as-grown state (no magnetic field applied during growth) is shown in Fig. 1(b). Here a single white domain in the Py traverses the Co islands. The magnetic spin structures are identical in both layers and it appears in Fig. 1(b) that the domain in the Py is responsible for the magnetic spin configuration in the Co. In order to identify the roles of the soft and patterned hard layers in determining the resulting magnetic spin configurations, we have carried out a detailed study of the magnetization reversal in similar systems, simply with arrays of shorter, $1.7 \times 5 \mu \mathrm{m}^{2}$, Co islands (see schematic of array geometry in the inset of Fig. 2). In order to facilitate our comprehension of the reversal processes, an external magnetic field of 300 Oe was applied parallel to the long axis of the islands during the deposition of the bilayer in order to set the orientation of the easy axis (shown in the inset of Fig. 2). Easy and hard axis hysteresis loops were measured using the MOKE on continuous 2 and $5 \mathrm{~nm}$ Py films (several $\mathrm{mm}^{2}$ areas) and continuous $2 \mathrm{~nm} / 2 \mathrm{~nm}$, $5 \mathrm{~nm} / 2 \mathrm{~nm}$, and $5 \mathrm{~nm} / 1 \mathrm{~nm}$ Py/Co bilayer films (1 Downloaded 10 Oct 2006 to 129.129.129.130. Redistribution subje $\times 1 \mathrm{~mm}^{2}$ areas). The films display typical hard and easy axis behavior with the easy axis coercivity $H_{c}=2$ and 3 Oe for the continuous Py films and Py/Co bilayers, respectively. The anisotropy fields $H_{k}$ are 6 and 3 Oe for the continuous 5 and $2 \mathrm{~nm}$ Py films, respectively, and $H_{k}=20$ Oe for the continuous Py/Co bilayers. We investigated the magnetization reversal in the patterned films with the magnetic field applied parallel to the easy axis and the corresponding hysteresis loops are given in Fig. 2. The hysteresis loops of the patterned bilayers with $5 \mathrm{~nm}$ Py films have classic easy axis square shapes with a small increase in coercivity as the Co thickness increases: $H_{c} \quad(1 \mathrm{~nm} \mathrm{Co})=4 \mathrm{Oe}$, and $H_{c}$ $(2 \mathrm{~nm} \mathrm{Co})=6$ Oe. However, for the $2 \mathrm{~nm}$ Py/2 nm Co patterned bilayer, the easy axis hysteresis loop shows a two stage switching process with two switching fields, $H_{c 1} \approx 6$ Oe and $H_{c 2} \approx 14$ Oe.

The differences in the easy axis switching behavior observed in the MOKE hysteresis loops can be elucidated by observing the differences in the magnetic spin configurations during reversal and typical domain configurations are shown in Fig. 3. We distinguish three regions in the continuous Py film within the area delimited by the island array: the Py located below the $\mathrm{Co}$ in the Py/Co islands, the Py present along the rows of islands (A), and the Py in the interisland regions (B). For the $5 \mathrm{~nm} \mathrm{Py/2} \mathrm{nm} \mathrm{Co} \mathrm{patterned} \mathrm{bilayer,}$ reversal occurs first in the Py layer before the Py/Co islands switch so that along the rows of islands [A in Fig. 3(a)] the spins in the continuous Py layer alternate, pointing up and down, with domain walls positioned at the island edges. In the presence of this regular array of unswitched Py/Co islands, the minimization of the local magnetic energy results in the formation of vertical and diagonal domains in the Py running between the islands [see interisland regions B in Fig. $3(\mathrm{a})$. These magnetic configurations are reminiscent of the magnetic spin structures observed in antidot arrays ${ }^{12}$ although, in contrast to arrays of holes in continuous films, the elongated Py/Co islands have a directionality, i.e., the magnetic spins in the surrounding Py prefer to lie parallel to the spins in the Py/Co islands rather than parallel to the shape borders. In addition, this direction can be changed with the applied magnetic field. At higher magnetic fields, the regions of reverse magnetic spins grow [Fig. 3(b)] and whisker domains are formed in the surrounding Py beyond the ends of the islands, reminiscent of those seen in antidot arrays. ${ }^{12}$ The reversal of the Py/Co islands is initiated at higher magnetic fields and occurs via propagation of domain walls nucleated near the ends of the islands [see white domains nucleated in the top row A in Fig. 3(a)]. In Fig. 3(b) it can be seen that two Py/Co islands have switched and their influence is "turned off" so that the domains in the neighboring Py disappear.

A very similar reversal (not shown) is observed in $2 \mathrm{~nm}$ Py/2 nm Co patterned bilayers although the Py/Co islands switch at a significantly larger magnetic field, resulting in the two stage switching observed in the hysteresis loop in Fig. 2. It is likely that the Py/Co islands have a higher switching field when the Py thickness is reduced from 5 to $2 \mathrm{~nm}$ because nucleation and depinning of domain walls cost more energy at lower thicknesses. ${ }^{13}$

When thinner Co islands $(1 \mathrm{~nm})$ are deposited on the $5 \mathrm{~nm}$ Py film, a strikingly different magnetization reversal is observed [Figs. 3(c) and 3(d)] which involves several different processes. Within the island array, reversal of the Py to AIP license or copyright, see http://apl.aip.org/apl/copyright.jsp 

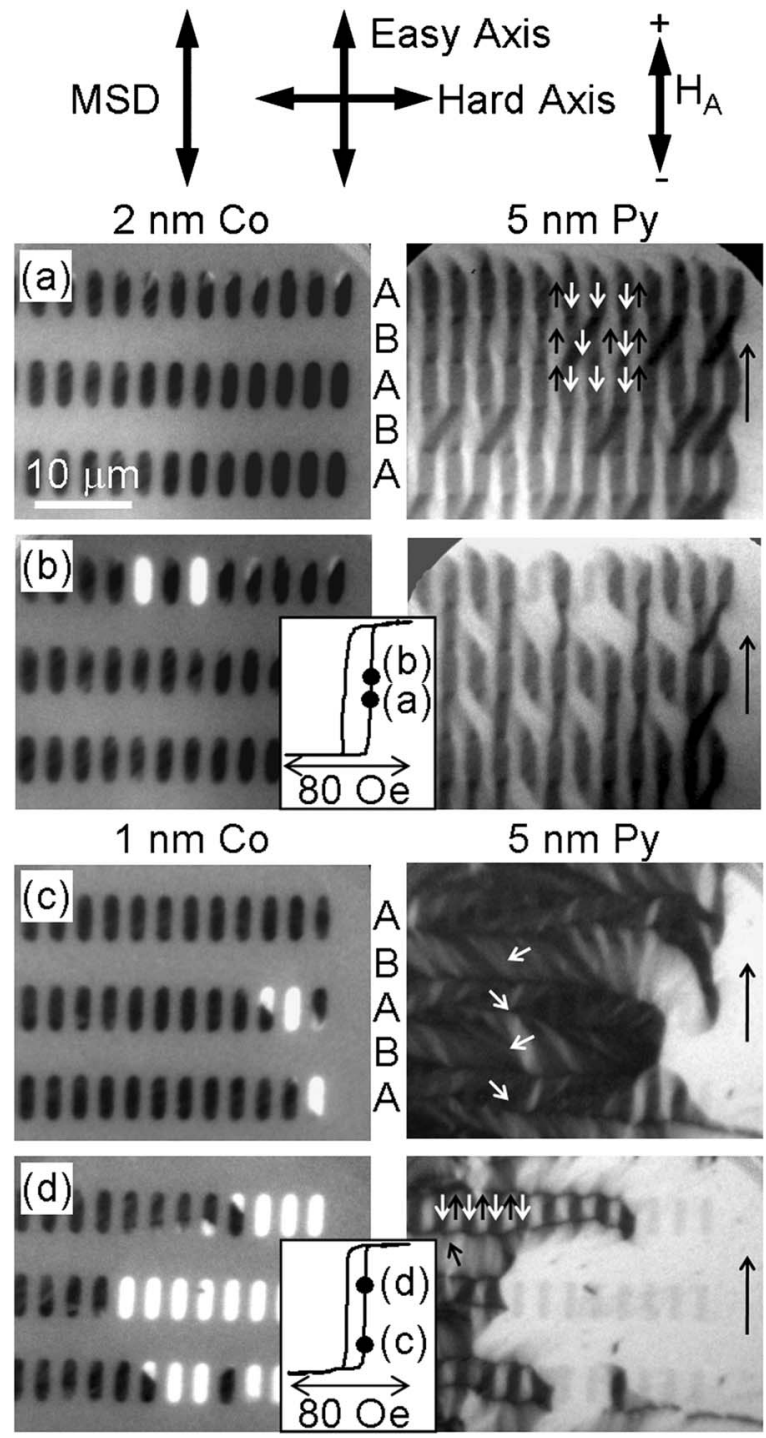

FIG. 3. Element-specific magnetic spin configurations at two different stages of the magnetization reversal in Py/Co bilayers with arrays of $5 \mu \mathrm{m}$-long Co islands (see inset of Fig. 2). The films were first saturated with a negative magnetic field $\left(H_{A}=-380 \mathrm{Oe}\right)$, which was subsequently reduced to zero. The images are taken as the magnetic field is increased in the positive direction and the corresponding position on the hysteresis loop is indicated in the insets. A indicates the row of islands and B is the interisland region. The arrows indicate the orientation of the magnetic spins.

initially occurs by rotation of the magnetic spins in bands which correspond to the rows of islands, A, and the interisland regions, B. Note that the white arrows in Fig. 3(c) represent the general orientation of the rotating magnetic spins which is orthogonal to the magnetization ripple contrast as expected in magnetic thin films. This behavior is reminiscent of coherent rotation during hard axis reversal in a magnetic thin film and implies that the rows of islands give rise to a quasi-easy-axis parallel to the rows. As the magnetic field is increased further [Fig. 3(d)], the spins in the Py continue to rotate towards the applied field while the spins in the Py/Co islands are hindered by the higher uniaxial stray field energy associated with the island shape. This again results in alternating spins in the Py, up and down, along the rows of islands and domain walls at the island boundaries. The magnetic spins in the Py rotate in opposite senses in bands A and B producing $360^{\circ}$ walls ${ }^{14}$ between the bands. These appear as horizontal dark lines between the ends of the islands and give a domain configuration that looks like ladders. Outside the island region, a large reverse domain appears in the Py and, as this region grows, the Py/Co islands switch as the domain wall in the Py sweeps past them. Once the domain boundary has passed by, the $360^{\circ}$ walls are mostly annihilated although, because of their very stable spin configuration, ${ }^{14}$ some wall sections remain even after the majority of reversal has occurred. Although the switching field of the $5 \mathrm{~nm} \mathrm{Py} / 1 \mathrm{~nm}$ Co patterned bilayer is similar to that of the $5 \mathrm{~nm} \mathrm{Py} / 2 \mathrm{~nm}$ Co bilayer, the stray field energy associated with the islands for the thinner Co layer is only sufficient to modify the reversal in the Py film but not to give the vertical and diagonal domains seen in Fig. 3(a) and is not able to resist the switching of the islands as the domain wall sweeps through the Py.

In conclusion, we have developed a method for the fabrication of magnetic islands of one ferromagnetic material exchange coupled to a continuous film of a second material with different ferromagnetic properties. When the patterned islands are harder than the continuous film, they modulate the behavior of the continuous film and allow the positioning of domain walls. The detailed reversal behavior in such bilayer systems, which cannot be interpreted from the MOKE hysteresis loops alone, depends critically on the magnetic properties of the films, which are in turn dependent on the film thickness. For the systems studied here, this determines whether the magnetization reversal occurs via the formation of vertical and diagonal domains or by a combination of coherent rotation and movement of a large domain boundary in the continuous film. These systems extend the possibilities for engineering magnetic thin film properties and could be of interest for positioning of domain walls in devices.

Part of this work was performed at the Swiss Light Source, Paul Scherrer Institut, Villigen, Switzerland. Work at Argonne was supported by the U.S. Department of Energy BES-MS, Contract No. W-31-109-ENG-38. Work at EPFL was partially supported by the Swiss Federal Office for Education and Science (OFES) in the framework of the ECfunded project NaPa, Contract No. NMP4-CT-2003-500120.

${ }^{1}$ B. D. Terris and T. Thomson, J. Phys. D 38, R199 (2005).

${ }^{2}$ J.-G. Zhu, Y. Zheng, and G. A. Prinz, J. Appl. Phys. 87, 6668 (2000).

${ }^{3}$ D. A. Allwood, G. Xiong, C. C. Faulkner, D. Atkinson, D. Petit, and R. P. Cowburn, Science 309, 1668 (2005).

${ }^{4}$ J. S. Jiang, J. E. Pearson, Z. Y. Liu, B. Kabius, S. Trasobares, D. J. Miller, S. D. Bader, D. R. Lee, D. Haskel, G. Srajer, and J. P. Liu, Appl. Phys. Lett. 85, 5293 (2004).

${ }^{5}$ E. E. Fullerton, J. S. Jiang, and S. D. Bader, J. Magn. Magn. Mater. 200, 392 (1999).

${ }^{6}$ H. Zeng, J. Li, J. P. Liu, Z. L. Wang, and S. Sun, Nature (London) 420, 395 (2002)

${ }^{7}$ D. Suess, T. Schrefl, S. Fähler, M. Kirschner, G. Hrkac, F. Dorfbauer, and J. Fidler, Appl. Phys. Lett. 87, 012504 (2005).

${ }^{8}$ D. McGrouthera and J. N. Chapman, Appl. Phys. Lett. 87, 022507 (2005).

${ }^{9}$ S. P. Li, W. S. Lew, J. A. C. Bland, L. López-Díaz, M. Natali, C. A. F. Vaz, and Y. Chen, Nature (London) 415, 600 (2002).

${ }^{10}$ J. Swerts, K. Temst, M. J. Van Bael, C. Van Haesendonck, and Y. Bruynseraede, Appl. Phys. Lett. 82, 1239 (2003).

${ }^{11}$ G. M. Kim, M. A. F. Van den Boogaart, and J. Brugger, Microelectron. Eng. 67-68, 609 (2003).

${ }^{12}$ L. J. Heyderman, F. Nolting, and C. Quitmann, Appl. Phys. Lett. 83, 1797 (2003).

${ }^{13}$ L. J. Heyderman, S. Czekaj, F. Nolting, E. Müller, P. Fischer, P. Gasser, and L. López-Díaz, J. Appl. Phys. 99, 063904 (2006).

${ }^{14}$ L. J. Heyderman, H. Niedoba, H. O. Gupta, and I. B. Puchalska, J. Magn. Magn. Mater. 96, 125 (1991). 